East African Medical Journal Vol. 85 No. 12 December 2008

LAPAROSCOPIC CONSERVATIVE MANAGEMENT OF A SPONTANEOUSLY RUPTURED SPLEEN: CASE REPORT

E. Opondo, MBChB, MMed (Surg), Consultant Surgeon and Lecturer, Jomo Kenyatta University of Agriculture and Technology, P. O. Box 62000-00200, Nairobi, Kenya and M.R. Khan, MD, PhD (Surg), Consultant Surgeon, Kenyatta National Hospital, Department of Surgery, P. O. Box 19625-00202, Nairobi, Kenya

Request for reprints to: Dr. E. Opondo, Institute of Tropical Medicine and Infectious Diseases, Jomo Kenyatta University of Agriculture and Technology, P. O. Box 62000-00200, Nairobi, Kenya

\title{
LAPAROSCOPIC CONSERVATIVE MANAGEMENT OF A SPONTANEOUSLY RUPTURED SPLEEN: CASE REPORT
}

\author{
E. OPONDO and M.R. KHAN
}

\begin{abstract}
SUMMARY
A case of an acute abdomen secondary to a ruptured spleen managed laparoscopically is presented. Laparoscopy is currently widely used for the initial diagnosis and management of such cases as an alternative to laparotomy due to the numerous advantages of the minimal access techniques. With widespread availability and adequate training in laparoscopy, minimal access surgery is likely to play a bigger role in the initial management of patients with an acute abdomen in Kenya. It is with this realisation that we present the case of a 25 year old male who presented with an acute abdomen and thrombocytopaenia due to a ruptured spleen following severe malaria that was managed laparoscopically. The aim is to highlight and encourage conservative management of acute abdomen due to splenic rupture in a patient with malaria.
\end{abstract}

\section{INTRODUCTION}

The spleen plays an important role in areas where malaria is endemic by producing antibodies against malaria parasites. Involvement of the spleen in malaria renders it more prone to complications such us enlargement and spontaneous rupture (1-8). Spontaneous rupture of the malaria infected spleen is rare due to the fact that it is tougher than the usual spleen. Prior to the 1970s treatment of a spontaneously ruptured spleen was by spleenectomy. Today $90 \%$ of the blunt paediatric splenic injuries and about 60 to $70 \%$ of the adult ones are managed conservatively (3).

We report a case of a spontaneously spleen presenting as an acute abdomen. Laparoscopy confirmed haemoperitoneum with a ruptured spleen that was managed conservatively. We present this case against the background that Kenya is a malaria endemic area and therefore more clinicians in this country are likely to manage such cases and should consider our approach. Modern laparoscopy was pioneered in Kenya by workers at the Aga Khan University Hospital, Nairobi and is used for diagnostic and therapeutic purposes $(9,10)$. The procedures currently done locally include cholecystectomy, appendicectomy, herniorraphy, total laparoscopic hysterectomy, and many other diagnostic procedures $(9,10)$. Laparoscopy definitely offers advantages of minimising surgical trauma and hence less systemic inflammatory reactions associated with conventional laparotomy. To date there is no reported case in the literature of spontaneously ruptured spleen managed laparoscopically as a complication of severe malaria in Kenya.

\section{CASE REPORT}

A 25 year old Somali male was admitted on $21^{\text {st }}$ May 2007 to The Mater Hospital, Nairobi with complains of severe abdominal pain, vomiting, and bloody diarrhoea lasting three days. He reported insidious onset of the above symptoms starting with a global headache and fever one week prior to admission. He had been treated at a local clinic in Eastleigh with antimalarial drugs that he could not remember. Three days prior to admission he developed bloody diarrhoea and haematuria despite treatment. While vomiting he developed sudden severe generalised abdominal pains. He had sought treatment at the Kenyatta National Hospital where he was diagnosed after blood tests and abdominal ultrasound to have malaria with a differential diagnosis of tuberculous peritonitis. He had been treated prior to this on several occasions for malaria at local clinics. He was a long distance truck driver who had returned from Sudan on $5^{\text {th }}$ August 2007.

On physical examination he was sick looking, pale, dehydrated and slightly jaundiced. He had no clinical features of chronic liver disease. Abdominal 
examination revealed generalised tenderness with board like rigidity and reduced bowel sounds. Examination of the other systems revealed normal findings.

Haematological findings on haemogram done on $20^{\text {th }}$ August 2007 were a haemoglobin level of $13.6 \mathrm{gm} / \mathrm{dl}$. WBC of $10.0 \times 10^{9} / 1$, RBC of $4.63 \times 10^{12}$ $/ 1$ and platelets of $40 \times 10^{9} / 1$. A repeat haemogram on $20^{\text {th }}$ August 2007 revealed significant changes to the previous findings with haemoglobin of $9.5 \mathrm{~g} / \mathrm{dl}, \mathrm{WBC}$ of $8,2 \times 10^{9} / 1$. RBC of $3.3 \times 10^{12} / 1$ and platelets of $28 \times 10^{9} / 1$. The peripheral blood film revealed thrombocytopaenia, and red blood cell polychromasia. Liver function tests revealed elevated total billirubin (193.20 uMol/1) and direct billirubin (90.30 uMol/1) while the liver enzymes were slightly elevated (AST-52.0 U/1 and ALT-53.9 U/1).

The widal test, urinalysis, and stool tests were all normal. Elisa test for human immunodeficiency virus wasnegative. Abdominal computerised tomography (CT Scan) reported a finding of a large pelvic ascites with a differential diagnosis of tuberculous peritonitis.

He was admitted with an impression of severe malaria and an acute abdomen. He was then started on intravenous quinine and also transfused one pint of whole blood. Surgical opinion was sought due to the severe abdominal pains and the diagnosis of an acute abdomen. Upon review a decision was made to do a diagnostic laparoscopy. He was then prepared for surgery as an emergency with two pints of blood on standby.

In theatre under general anaesthesia laparoscopy was performed with a pneumoperitoneum of $15 \mathrm{mmHg}$ created to allow for visualisation of the peritoneal cavity by introduction of carbon dioxide under controlled pressure. This was done by direct visualisation with placement of a $10 \mathrm{~mm}$ working laparoscope with a video monitor.

Laparoscopic findings were haemoperitoneum with a ruptured spleen and an adherent clot in the sub diaphragmatic space. An additional $5 \mathrm{~mm}$ port was placed in the right upper quadrant with introduction of a grasping forceps and probes introduced in turns to explore the peritoneal cavity further. The spleen had a single tear approximately $3 \mathrm{~cm}$ along the superior surface with an attached adherent clot. The liver, gallbladder, stomach, colon, appendix and small intestines were found to be normal after peritoneal lavage and suction of blood from the peritoneal cavity was done. No surgical intervention was done as the adherent clot was found to be providing tamponade with no active bleeding evidenced. A closed drain was left in situ through the $5 \mathrm{~mm}$ port. Post operatively he improved remarkably and continued on anti-malarials, haematinics and bed rest. He was discharged on the fifth day post operative to continue with strict bed rest at home. He remains stable on subsequent follow up in the clinic.

\section{DISCUSSION}

Pathological rupture of the spleen occurs in malaria, kala-azar, infectious mononucleosis, typhoid, amyloidosis, and gaucher's disease (8). Plasmodium parasites are highly specific, with humans as the only vertebrate hosts and anopheles mosquitoes as the vectors. Accurate information on the global incidence of malaria is difficult to obtain because of poor reporting in areas where malaria is endemic like in sub-Saharan Africa. The global incidence of malaria is estimated to be nearly 120 million clinical cases per year with about 300 million people acting as disease carriers.

The majority of malaria complications occur on the African continent and in 1991 the World Health Organisation estimated 800,000 deaths per year among African children (8). The incidence of splenic rupture in complicated malaria is poorly defined. Fifteen cases of splenic rupture have been reported in world literature since $1960(2,4-6)$. This suggests the possibility of under reporting or under diagnosis especially in areas like Africa where malaria is endemic.

Splenic enlargement is found in 50 to $80 \%$ of populations where malaria is endemic. When a palpable spleen is present it is generally appreciated within three to four days after onset of disease symptoms. Plasmodium vivax has been the species most associated with splenic rupture (6). Spontaneous rupture occurs most frequently in the acute phase and during the primary illness (6). This patient had enlargement that also caused thrombocytopaenia leading to haematuria and haematochezia. He sustained splenic rupture during the acute phase of this illness despite having had previous episodes in the past.

The exact mechanism of splenic rupture is unknown but three possible mechanisms have been described (3). The first is an increase in intrasplenic tension that is due to cellular hyperplasia and engorgement. Secondly the spleen maybe compressed by abdominal musculature during physiological activities such as sneezing, coughing defecation and sitting up or turning in bed. This seems to have been the mechanism in this patient because he experienced sudden severe abdominal pains after a bout of vomiting. The final mechanism that has been attributed is vascular occlusion due to reticuloendothelial hyperplasia resulting 
in thrombosis and infarction. This then leads to interstitial and subcapsular haemorrhage. The distended capsule finally gives way.

Pathological findings in spontaneous splenic rupture include gross and microscopic changes. On gross examination the spleen is usually dark red in colour because of hyperaemia and deposition of haemozin (malarial pigment). The capsule is usually thin and friable. The tear is usually single and on any splenic surface but multiple tears has been reported. Microscopic examination normally reveals diffuse hyperplasia with sinusoids showing subintimal adventitial leucopoiesis and thrombosis $(7,8)$.

Spleenectomy is the widely accepted mode of treatment in cases of spontaneously ruptured spleen. But due to the known importance of the spleen in immune response to pneumococcal and malaria infections spleenectomy should be reserved for those patients with severe rupture or those with continued or recurrent bleeding on conservative management. In conservativemanagement thepatient'shaemodynamic status is maintained and strict bed rest instituted. Repeat ultrasonography or computed tomography is done to assess the healing process.

The realisations that patients without a spleen have an increased risk of death from overwhelming post spleenectomy sepsis lead surgeons in America and Europe to consider methods of splenic conservation (3). Initially operative repair and later splenorrhaphy were attempted but with introduction of the CT Scan non-operative management became popular and later predominant (3). Non-operative protocols initially required a strict criterion of haemodynamic stability, isolated splenic lesions, low grades of CT Scan defined lesions and conscious patients (for assessment of peritonitis). These indications have been broadly expanded especially in dedicated trauma centres with readily available theatre and intensive care monitoring. It will be important to note the role that laparoscopy will play in conservative management options as there is limited literature at the moment on this. The obvious indication is likely to be those patients who will be noted to have peritonitis and thrombocytopaenia like in this case report. It is however important to note the possible risks of non-operative management like delayed haemorrhage, splenic pseudocyst, obsesses and pseudo aneurysms (1-3). Interestingly the value of follow up imaging has not been established and recommendations on resumption to full time activity except contact sports have been reduced to grade of injury plus two weeks (11).
In Kenya and the African continent logistical issues limit the advisability of conservative management especially monitoring requirements like in house surgical capability, rapid theatre mobilisation and transfusion services.

In conclusion we wish to advocate for conservative management of the spontaneously ruptured spleen in settings where haemodynamic stability can be maintained and imaging tests to assess healing readily available.

\section{ACKNOWLEDGEMENTS}

To the Medical Director of The Mater Hospital and to the Standards and Ethics Committee for giving permission to publish this case.

\section{REFERENCES}

1. Hamilton, D.R. and Douglas, P. Ruptured spleen in a malarious area: with emphasis on conservative management in both adults and children. Aust. NZ. J. Surg. 1982; 52: 310-313.

2. Clezy, J.K.A. and Richens, J.E. Non-operative management of spontaneously ruptured spleen. $\mathrm{Br}$. J. Surg. 1985; 72: 990.

3. Upadhyaya P. Conservative management of splenic trauma: History and current trends. Paediatr. Surg. Int. 2003; 34: 137-174.

4. Davis, R. Spontaneous rupture of the pathological spleen in malaria. S. Afr. Med. J. 1973; 47: 1801-1803.

5. Mokashi, A.J., Shirahati, R.G., Prabhu, S.K., et al. Pathological rupture of a malarial spleen. Postgrad. Med. 1992; 38: 141-142.

6. Zigman, B.S. and Viner, B.L. Splenic complications in malaria: Case report. Clin. Infect. Dis. 193; 16: 223-232.

7. Shwartz, S.I. Spleen principles of surgery, Edn 5. Edited by Shwartz S.I.Shires G.T and Spencer: $M c$ Graw-Hill, 1989; 1445-1457.

8. Lubiz, J.M. Pathology of the ruptured spleen in acute vivax malaria. Blood. 1949; 4: 1168-1175.

9. Patel, S.C .and Bhatt, J.R. Laparoscopic cholecystectomy at the Aga Khan Hospital Nairobi. East Afr. Med. J. 2000; 77: 197-198.

10. Baraza, R. Laparoscopic cholecystectomy at the Nairobi Hospital: A personal experience with 42 cases. East Afr. Med. J. 2005; 82: 473-476.

11. Styllianos, S. Evidence-based guidelines for resource utilization in children with isolated spleen or liver injury. Paediat. Surg. 2000; 35: 164-169. 